\title{
Decision Making, Bias, and Low Grade Glioma
}

Can J Neurol Sci. 2011; 38: 193-194

We all face decisions on a daily basis. The choices we make not only affect us, but also reflect much about us. There have been studies in the psychology and neurobiology literature about cognitive decision making ${ }^{1,2}$, a few studies on how physicians make decisions for patients ${ }^{3}$, however these issues have rarely been studied from the patient's perspective. Decision making regarding course of treatment where the outcome is uncertain is even more trying for an already stressed patient ${ }^{4}$. Often patients use intuitive feelings and simple decision skills to guide them in such situations.

Consider the case of low grade gliomas - a dilemma that is yet to be solved. How do patients come to a treatment decision, and specifically, why do many patients feel an intuitive need for surgery? Surgical resection of WHO I gliomas such as pilocytic astrocytoma, is clearly beneficial, however the value of removal of more infiltrative gliomas is not so clear and remains the subject of controversy ${ }^{5,6}$. Early resection may cause neurological impairment ${ }^{5-7}$, when symptoms can often be controlled with medications until tumor progression is documented. Physicians' and surgeons' opinions on management range from early surgical resection to observation until surgery (and adjuvant treatment) becomes necessary. Patients, however, may succumb to decision processing errors, and make the assumption that surgery is their best option regardless of their specific case. This intuitive decision can be attributed to the following factors: (1) framing effects and presentation; (2) poor cognitive understanding; and (3) various biases. The case of low grade gliomas represents an exemplary situation in which a decision must be made under uncertainty, where even the doctor does not hold the absolute answer.

Our interpretation and assessment of a situation strongly depends on the way it is framed, or presented. Differences in how a situation is framed have been shown to result in contradictory perceptions and choices. People are often unaware of how a change in formulation of a situation changes their perspective $^{8}$. In one study, data from results of surgery and radiation therapy for lung cancer were presented to a population of 238 ambulatory patients, 491 graduate students and 424 physicians ${ }^{9}$. The situation was framed in two different ways - in terms of mortality rate, and of survival rate. The study found that the appeal of surgery compared to radiation was significantly higher when framed in terms of survival. Experienced physicians were strongly affected by the framing of this situation as well as patients. Clearly, framing effects can produce a bias which can affect the way patients perceive information which they use to make decisions. Intuitive decisions that advocate for surgery may be a result of a framing that minimizes the importance of related risks and consequentially maximizes the appeal of surgery. In diseases like low grade glioma, patients who are dealing with decisions under uncertainty may be even more susceptible to framing effects. Avoiding framing effects can be particularly difficult, but in order to fully respect patient autonomy, it is important for physicians to be able to communicate clearly without bias.

Informed consent proposes that competent patients understand information and strive to make an autonomous and optimal decision regarding their treatment. However, to what extent are people able to truly comprehend complex medical information without any preconceived notions ${ }^{10}$ ? It is easy to subconsciously favour surgery over observation by underestimating the associated risks and undervaluing the benefits of observation, the "wait and see" approach. Increasing the patient's knowledge of the related risks of surgery may reach beyond percentage probabilities. Use of quantitative, qualitative and graphic/visual formats can help patients better comprehend risks $^{11}$. In addition to poor risk perception, patients who intuitively feel that surgery is their best option may have formed a preconceived notion that action is less dangerous then inaction. Patients with low grade gliomas may also feel anxious and fearful that they are in a time-sensitive situation, therefore some may feel as if the "wait and see" approach is counterproductive or even dangerous. People tend to desire solutions that fully eliminate a risk rather than reduce $i^{12}$, which is why in order to realistically approach the decision to undergo surgery, the patient must have a full understanding that surgery for low grade glioma has risks, is not usually a definitive cure, and that tumor recurrence is a strong possibility.

In some instances, even when the patient has an understanding of the risks and benefits of surgery, and knows that a course of observation is an option, the conviction to undergo surgery may be overpowering. This can be attributed to various biases that do not always have a rational foundation. An optimism bias that one is less at risk for danger than others ${ }^{13}$ can cause patients to overlook the associated risks and seek surgical intervention. Similarly, an availability bias can also be an important factor that influences patients to seek surgery without rational reasoning to support their decision. The availability bias suggests that people overestimate situations and risks that have received widespread attention ${ }^{14-16}$, because it is more available in their memory. Consider the recent Swine flu pandemic. It is estimated that between 8870 and 18300 deaths occurred between April 2009 and April 2010 in the United States ${ }^{17}$. The H1N1 outbreak was a highly publicized event, and was widely feared. Rationally, the same fear should be applied to common influenza, which claims an estimated 36000 Americans each year $^{18}$, but the common flu was relegated to the background during the H1N1 pandemic.

The notoriety that brain tumors have attained in the public eye, similar to the H1N1 outbreak, can evoke fear in patients who in turn develop a compelling urge to take aggressive action against what they may see as a highly dangerous situation. Physicians can help ease such irrational deductions by 
communicating to patients that there is such an entity as an indolent low grade glioma where "wait and see" is safe, progression of the tumor may be many years away, and sometimes surgery can be more of a risk than a benefit. We are all susceptible to our own biases, and they are not always voluntary.

Decisions regarding a course of treatment are the most difficult to make when this decision needs to be made under conditions of significant uncertainty. In such positions, patients may often express views as a result of framing and personal biases. Empiric and intuitive deductions will not always yield a completely rational decision, which is why patient-physician interactions are most important in such situations. Depending on some patients' personalities, surgery may feel like the right option and competent patients ultimately have the right to choose their own course of treatment. To successfully meet the expectations of informed consent and respect for patient autonomy, an understanding of the common errors that patients are prone to in decision-making can be beneficial for physicians, and ultimately patients as well.

We are not recommending that every low grade glioma patient be managed with the "wait and see" approach. Early and aggressive surgery may be beneficial to outcome, and also help some patients psychologically, although we have no Class I evidence to support this. We are recommending that physicians and surgeons continue to strive to educate our patients as best we can so they can make the right decision for themselves, with the minimum of bias from within themselves. An integral part of that begins with we doctors striving for a minimum of bias and dogma on our part when we convey pertinent information to our patients who are in medically uncertain situations.

Praveena Deekonda, Mark Bernstein Toronto Western Hospital Toronto, Ontario, Canada

\section{REFERENCES}

1. Ernst N, Paulus P. Neurobiology of decision making: a selective review from a neurocognitive and clinical perspective. Biol Psychiatry. 2005; 58:597-604.

2. Fellows L. The cognitive neuroscience of human decision making: a review and conceptual framework. Behav Cogn Neurosci Rev. 2004; 3:159-72.

3. Aliyama Y, Houkin K, Nozaki K, Hashimoto N. Practical decisionmaking in the treatment of unruptured cerebral aneurysm in Japan: The U-CARE study. Cerebrovasc Dis. 2010; 30:491-9.

4. Politi MC, Clark MA, Ombao H, Dizon D, Elwyn G. Communicating uncertainty can lead to less decision satisfaction: a necessary cost of involving patients in shared decision making? Health Expect. Epub 2010 Sept. 23.

5. Bampoe J, Bernstein M. The role of surgery in low-grade glioma. J Neuro-Oncol. 1999; 42:259-69.

6. Smith JS, Chang EF, Lamborn KR, et al. Role of extent of resection in the long-term outcome of low-grade hemispheric gliomas. J Clin Oncol. 2008; 26:1338-45.

7. Khu KJ, Ali S, Bernstein M. Conversion disorder after surgery in a brain tumour patient. Can J Neurol Sci. 2010; 37:293-5.

8. Tversky A, Kahneman D. The framing of decisions and the psychology of choice. Science. 1981; 211:453-8.

9. McNeil BJ, Pauker SG, Sox, HC, Tversky A. On the elicitation of preferences for alternative therapies. N Engl J Med. 1982; 306: 1259-62.

10. Bernstein M. Fully informed consent is impossible in surgical clinical trials. Can J Surg. 2005; 48(4):271-2.

11. Schapira MM, Nattinger AB, McAuliffe TL. The influence of graphic format on breast cancer risk communication. J Health Commun. 2006; 11:569-82.

12. Redelmeier DA, Rozin P, Kahneman D. Understanding patient's decisions: cognitive and emotional perspectives. JAMA. 1993; 270:72-6.

13. Weinstein ND. Optimistic bias about personal risks. Science. 1989; 246:1232-3.

14. Tversky A, Kahneman D. Availability: a heuristic for judging frequency and probability. Cognitive Psychol. 1973; 5:677-95.

15. Tversky A, Kahneman D. Judgment under uncertainty: heuristics and biases. Science. 1974; 185:1124-31.

16. Bogardus ST, Holmboe E, Jekel JF. Perils, pitfalls, and possibilities in talking about medical risk. JAMA. 1991; 281:1037.

17. Centre for Disease Control and Prevention. Updated CDC estimates of 2009 H1N1 influenza cases, hospitalizations and deaths in the United States, April 2009-April 10, 2010. CDC Web 2010. [cited 2010 Aug 2] Available at: http://www.cdc.gov/h1n1flu/ estimates_2009_h1n1.htm.

18. Sibbald B: Estimates of flu-related deaths rise with new statistical models. CMAJ Web 2003. [cited 2010 Aug 2] Available at: http://www.cmaj.ca/cgi/ content/full/168/6/761-a. 